\title{
Path Following Demonstration of a Hybrid Cable-Driven Parallel Robot
}

\author{
Micaël Michelin ${ }^{1}$, Pierre Elie Hervé ${ }^{1}$, Olivier Tempier ${ }^{2}$, Jean Baptiste Izard ${ }^{1}$ and \\ Marc Gouttefarde ${ }^{2}$ \\ ${ }^{1}$ TECNALIA, Basque Research and Technology Alliance, Montpellier, France \\ ${ }^{2}$ LIRMM, Univ Montpellier, CNRS, France \\ marc.gouttefardedirmm.fr
}

\begin{abstract}
In the context of applications requiring large workspace such as painting of airliners, a cable-driven parallel robot (CDPR) having a manipulator (robot arm) on-board its mobile platform possesses an interesting potential. Such a robotic system has typically more than six degrees of freedom (DOFs) and, hence, more than the minimum required to accomplish a task in Cartesian space. In particular, a robotic system consisting of a six-DOF cable-driven parallel robot whose mobile platform is equipped with a seven-DOF manipulator is considered in this paper. This robotic system being kinematically redundant, a redundancy resolution scheme is used to allow its end-effector to follow a relatively complex path in Cartesian space while avoiding cable collisions and satisfying some constraints on the CDPR workspace.
\end{abstract}

Keywords: Hybrid cable-driven parallel robot, kinematic redundancy

\section{Introduction}

Cable-Driven Parallel Robots (CDPRs) are known for their potentially very large workspace and heavy payload capabilities, e.g. [1, 2, 3, 4, 5, 6]. Manufacturing, manipulation, and inspection in various industries (e.g. aeronautic, naval, nuclear, construction, renewable energy) may require a local dexterity that the CDPR mobile platform alone cannot provide. Examples of such applications are painting or sandblasting [7] and assembly [8] of large parts. Therefore, in order to accomplish such tasks, it can be relevant to equip the mobile platform of a large-dimension CDPR with a manipulator (robot arm).

Placing an actuated mechanical device on-board the mobile platform of a CDPR is not a novel idea, e.g. [9, 10,11], but only few previous works deal with it. A kinematic analysis of a 3 degree-of-freedom (DOF) manipulator suspended on one cable is presented in [10]. In [12], a pendulum-like underactuated robot, consisting of a 1-DOF actuated pendulum suspended on a single cable, is introduced. Moreover, the works presented in $[13,14]$ deal with underconstrained CDPRs having a multiple DOF manipulator placed on their mobile platform. In [14], the static analysis of a planar 2-cable suspended CDPR having an on-board 4-DOF planar manipulator is presented. In [13], 
a 2-DOF planar manipulator is placed on a planar 2-cable suspended CDPR in order to improve the range of possible orientations along a trajectory. Hence, in these previous works, the CDPR is underconstrained which means here that the mobile platform is driven by less cables than its number of DOFs. In the present paper, the case of redundantly actuated 6-DOF CDPRs, i.e., CDPRs driven by more than six cables, is considered. A few previous works deal with redundantly actuated CDPRs equipped with a manipulator. A static analysis of planar 3-DOF suspended CDPRs with a manipulator on-board the mobile platform is proposed in [15]. Recently, in [16], the decoupled modeling, state estimation, and model-predictive vibration control of a kinematically-constrained redundant planar CDPR equipped with two 1-DOF pendulums are discussed.

When a 6-DOF CDPR mobile platform is equipped with a multiple DOF manipulator, the resulting Hybrid Cable-Driven Parallel Robot (HCDPR) is kinematically redundant. Hence, in order to generate feasible generalized joint coordinates allowing the HCDPR end-effector to follow a path, standard kinematic redundancy resolution or path planning techniques can be used. In the recent works [17, 18], continuous path validation methods integrated into the path planning software HPP [19] were proposed for the HCDPR consisting of the six-DOF CDPR prototype CoGiRo [3, 20] equipped with a seven-DOF manipulator Yaskawa SIA20F. In the present paper, the same HCDPR is considered but standard kinematic redundancy resolution techniques based on local optimizations are used to generate feasible generalized joint coordinates which allow the HCDPR end-effector to follow a path, while avoiding cable collisions and satisfying some workspace constraints. The results are validated in simulations and in experimental demonstrations [21,22] which form the contribution of this work since, to the best of our knowledge, such practical experimental results were never reported before.

This paper is organized as follows. Section 2 describes the HCDPR considered in this work. Section 3 presents the kinematic model while Section 4 introduces the kinematic redundancy resolution method used to solve the inverse kinematics. Finally, Section 5 presents the simulation and experimental results.

\section{HCDPR Description}

One concern when installing a manipulator on a large-dimension CDPR mobile platform is the electrical cable(s) required to bring power to the CDPR mobile platform. In the case of the CDPR prototype CoGiRo [3], whose global dimensions are $15 \mathrm{~m} \times 11$ $\mathrm{m} \times 6 \mathrm{~m}(\mathrm{Lx} 1 \times \mathrm{h})$, it is critical to integrate both the serial manipulator and its controller 


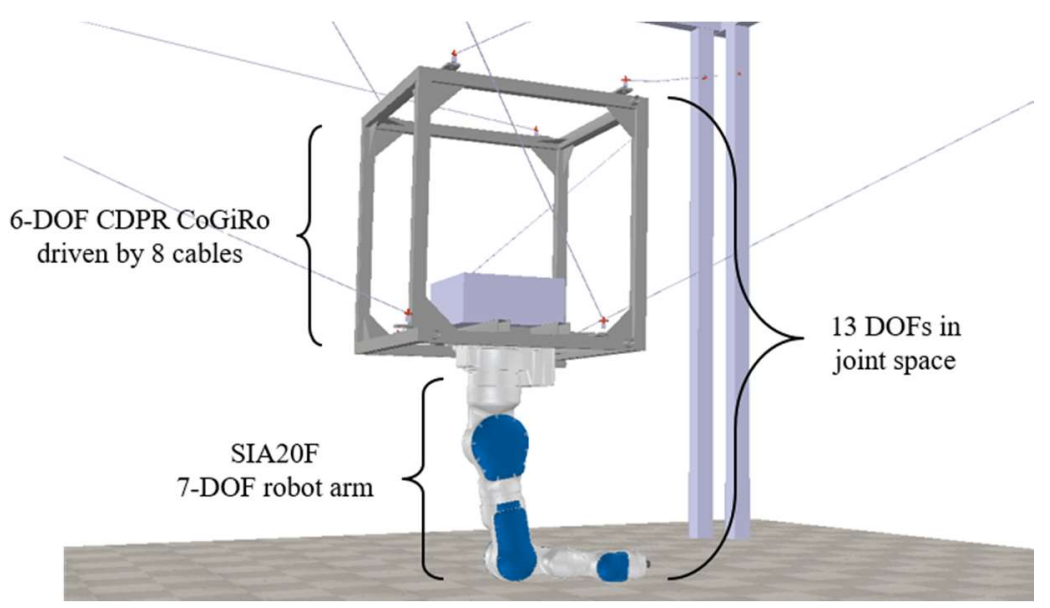

Fig. 1. The HCDPR consisting of the CDPR CoGiRo equipped with a Yaskawa SIA20F manipulator (shown as implemented in a V-REP simulator of the system).

on-board the mobile platform so that only one data link (Ethernet cable) and one power cable are required. The prototype CoGiRo is a suspended CDPR (all its cable drawing points are located on top of this working volume) and its mobile platform is a cube with a side length of $1 \mathrm{~m}$. The on-board manipulator should thus be placed below the mobile platform and should have a working range of around $1 \mathrm{~m}$. The maximum payload capability of CoGiRo across a large part of its wrench-feasible workspace being $500 \mathrm{~kg}$, the desired net mass of the on-board manipulator and its controller should not be larger than $300 \mathrm{~kg}$. Moreover, the manipulator payload capability should not be too limiting and its controller should have communication capabilities with external systems (here, the CDPR control system). We therefore set the main requirements on the choice of the manipulator to be placed on-board the CDPR mobile platform as follows:

- Manipulator payload capability: $20 \mathrm{~kg}$;

- Working range: $1 \mathrm{~m}$;

- Controller box fitting inside a $1 \mathrm{~m}$ side cube;

- Target point on-the-fly request capabilities with relatively small latency;

- Total mass (manipulator + controller box) lower than $300 \mathrm{~kg}$.

According to the requirements summarized above, the manipulator Yaskawa SIA20F was found to be a good choice. Its controller FS100 has a relatively low mass and can remotely receive on-the-fly target points, e.g. such as demonstrated by the capability of the available ROS compatible FS100 drivers to move the end-effector in Cartesian space using a gamepad [23]. The SIA20F is a 7-DOF anthropomorphic manipulator (S-R-S manipulator) with a rated payload of $20 \mathrm{~kg}$, a relatively low net mass (120 kg for the manipulator itself and $26 \mathrm{~kg}$ for the FS100 controller) and a working range of $1118 \mathrm{~mm}$ from the elbow central joint to the wrist flange. It also features a high torque capacity at the wrist allowing operations such as drilling, which is one of the objectives of the DexterWide project [24]. With the SIA20F manipulator and its controller on-board the mobile platform of the CDPR CoGiRo (Fig. 1), the platform of 
the resulting robotic system weighs a total of $296 \mathrm{~kg}$ (without payload at the end-effector of the SIA20F).

\section{$3 \quad$ Kinematic Model}

\subsection{Kinematic and actuation redundancy}

The robotic system shown in Fig. 1 is an HCDPR (a hybrid parallel/serial robot system) having two levels of redundancy. The CoGiRo CDPR has an actuation redundancy of degree two with eight driving cables for a six-DOF mobile platform. The manipulator SIA20F has one degree of kinematic redundancy with seven joints for six DOFs at its end-effector. The HCDPR has then fifteen actuated DOFs for six DOFs at the endeffector and possesses both actuation and kinematic redundancies. Methods allowing to manage these two types of redundancy are widely presented in the literature. For serial manipulators, a well-known method is to use an iterative solution of the inverse geometric problem based on the pseudo-inverse of manipulator Jacobian matrix. The generalization of this method with projection of joint velocities in the Jacobian nullspace allows to perform joint motions without any influence on the end-effector Cartesian pose. These "internal" joint motions can be used to achieve secondary tasks such as obstacle and joint limit avoidance, or maximizing manipulability, e.g. [25, 26]. Similar methods allow one to manage actuation redundancy of parallel robots using the transpose of the Jacobian matrix to control internal forces in the mechanical structure.

\subsection{Selection of generalized joint coordinates}

In fact, the methods presented [27] are currently used to solve the actuation redundancy of the CDPR CoGiRo and to control the motions of its mobile platform, the motion control being based on a controlled winding of the cables. Therefore, in the present paper, it is considered that the six DOFs of the mobile platform of the CDPR CoGiRo are properly controlled. Hence, the CDPR mobile platform DOFs can be considered directly in a purely kinematic redundancy resolution problem where the eight cable lengths are replaced by six variables defining the mobile platform pose:

$$
\left[\begin{array}{llllllll}
l_{1} & l_{2} & l_{3} & l_{4} & l_{5} & l_{6} & l_{7} & l_{8}
\end{array}\right]^{T} \rightarrow\left[\begin{array}{llllll}
x & y & z & \phi & \theta & \psi
\end{array}\right]^{T}
$$

The X-Y-Z Euler angle convention is used to define the three angles $\phi, \theta$, and $\psi$ which define the orientation of the mobile platform, while $x, y$ and $z$ define the position of the reference point of the mobile platform. Then, for the overall system consisting of the CDPR mobile platform and its on-board manipulator (with joint coordinates $q_{i}$ ), the following generalized (hybrid) coordinate vector $\boldsymbol{Q}$ is defined by concatenating the six mobile platform pose variables and the seven SIA20F joint coordinates:

$$
\boldsymbol{Q}=\left[\begin{array}{lllllllllllll}
z & y & x & \phi & \theta & \psi & q_{1} & q_{2} & q_{3} & q_{4} & q_{5} & q_{6} & q_{7}
\end{array}\right]^{T}
$$

The thirteen components of vector $\boldsymbol{Q}$ are considered as joint coordinates. The mobile platform of the CDPR can thus be viewed as a floating base allowing to position and 
orient the on-board manipulator base arbitrarily in Cartesian space (within the limits of the CDPR position and orientation workspaces).

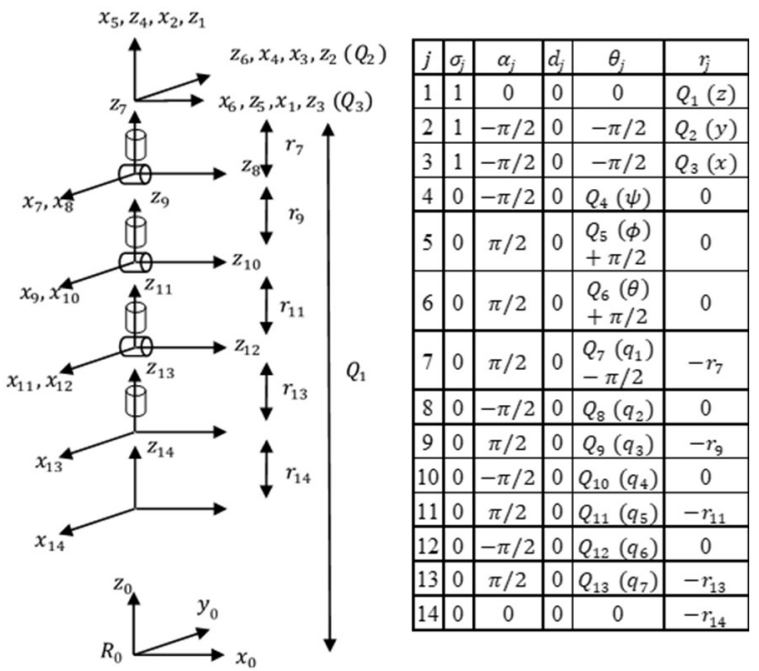

Fig. 2. Kinematic modeling as an equivalent serial chain (left) and corresponding Denavit-Hartenberg parameters (right).

\subsection{Serial kinematic chain and Denavit-Hartenberg parameters}

Based on Section 3.2, the overall system can be considered as a serial kinematic chain consisting of three prismatic joints and three revolute joints (platform of the CDPR CoGiRo), followed by seven revolute joints (manipulator SIA20F). The corresponding kinematic modeling and the Denavit-Hartenberg parameters are shown in Fig. 2 ( $\sigma_{j}=1$ for a prismatic joint and $\sigma_{j}=0$ for a revolute joint). Frame $R_{0}$ is a fixed reference frame and frame $R_{14}$ is the mobile frame attached to the HCDPR end-effector.

\section{$4 \quad$ Kinematic Redundancy Resolution}

For a kinematically redundant robotic systems such as the one under study, there exist infinitely many joint positions for a given end-effector pose. To deal with this redundancy, following the guidelines provided in $[25,26]$, an iterative solution of the inverse geometric problem of the serial kinematic chain introduced in Section 3 is presented in this section.

Iteratively solving the inverse geometric problem consists in computing the joint coordinate increments $d \boldsymbol{Q}$ corresponding to an end-effector pose increment $d \boldsymbol{X}$. Here, $\boldsymbol{X}$ is the manipulator end-effector pose vector with respect to the base frame $R_{0}$ and with Euler angles used to define the orientation:

$$
\boldsymbol{X}=\left[\begin{array}{llllll}
x_{e} & y_{e} & z_{e} & \phi_{e} & \theta_{e} & \psi_{e}
\end{array}\right]^{T}
$$


The increment $d \boldsymbol{X}$ is computed from the current end-effector pose $\boldsymbol{X}_{\boldsymbol{t}}$ and the desired one at the next sample period $\boldsymbol{X}_{\boldsymbol{t}+\mathbf{1}}$. These poses are first converted into the corresponding homogeneous transformation matrices relative to base frame $R_{0}$ :

$$
{ }^{0} \mathbf{T}_{\mathrm{t}}=\left[\begin{array}{cccc}
\boldsymbol{s}_{t} & \boldsymbol{n}_{t} & \boldsymbol{a}_{t} & \boldsymbol{P}_{t} \\
0 & 0 & 0 & 1
\end{array}\right], \quad{ }^{0} \boldsymbol{T}_{t+1}=\left[\begin{array}{cccc}
\boldsymbol{s}_{t+1} & \boldsymbol{n}_{t+1} & \boldsymbol{a}_{t+1} & \boldsymbol{P}_{t+1} \\
0 & 0 & 0 & 1
\end{array}\right]
$$

where $\boldsymbol{s}, \boldsymbol{n}$ and $\boldsymbol{a}$ are the columns of the corresponding rotation matrix and $\boldsymbol{P}$ is the position vector of the end effector in $R_{0}$. Then, the end-effector increment $d \boldsymbol{X}$ is given by:

$$
d \boldsymbol{X}=\left[\begin{array}{c}
\boldsymbol{P}_{t+1}-\boldsymbol{P}_{t} \\
\frac{1}{2}\left(\boldsymbol{s}_{t} \times \boldsymbol{s}_{t+1}+\boldsymbol{n}_{t} \times \boldsymbol{n}_{t+1}+\boldsymbol{a}_{t} \times \boldsymbol{a}_{t+1}\right)
\end{array}\right]
$$

The increment $d \boldsymbol{Q}$ can be obtained by means of the Jacobian matrix pseudo-inverse: $d \boldsymbol{Q}=\boldsymbol{J}^{+} d \boldsymbol{X}$ where $\boldsymbol{J}$ is the Jacobian matrix of the serial kinematic chain. This method brings forth the particular solution minimizing $\|\dot{\boldsymbol{Q}}\|^{2}$. Computing other solutions for $d \boldsymbol{Q}$ can classically be done by adding a term to $d \boldsymbol{Q}=\boldsymbol{J}^{+} d \boldsymbol{X}$ :

$$
d \boldsymbol{Q}=\boldsymbol{J}^{+} d \boldsymbol{X}+\left(\boldsymbol{I}_{n}-\boldsymbol{J}^{+} \boldsymbol{J}\right) \boldsymbol{Z}
$$

This second term allows the projection of any joint velocity vector $\boldsymbol{Z}$ into the Jacobian matrix null space, these joint velocities thus generate no displacement of the end-effector. This second term can be used to optimize a cost function $H(\boldsymbol{Q})$, for instance by computing $\boldsymbol{Z}=k_{H} \boldsymbol{\nabla} \boldsymbol{H}$ where $H$ is a scalar function depending on the joint variables, $H(\boldsymbol{Q})=F\left(Q_{1}, \ldots, Q_{13}\right)$ and $\boldsymbol{\nabla} \boldsymbol{H}$ is the gradient of $H(\boldsymbol{Q}), \boldsymbol{\nabla} \boldsymbol{H}=\left[\frac{\partial H}{\partial Q_{1}} \ldots \frac{\partial H}{\partial Q_{13}}\right]^{T}$. Equation (6) can then be written as the following well-known equation:

$$
d \boldsymbol{Q}=\boldsymbol{J}^{+} d \boldsymbol{X}+k_{H}\left(\boldsymbol{I}_{n}-\boldsymbol{J}^{+} \boldsymbol{J}\right) \boldsymbol{\nabla} \boldsymbol{H}
$$

When the scalar $k_{H}$ is positive, the cost function $H$ is maximized and when $k_{H}$ is negative, it is minimized. Function $H$ can express various objectives, for example the distance between a robot part and an environment object or the distance to joint limits. These functions may thus be used to avoid collisions and joint limits. Moreover, several optimization functions $H_{i}$ and associated scalars $k_{H i}$ can be considered in order to include several objectives into the problem:

$$
d \boldsymbol{Q}=\boldsymbol{J}^{+} d \boldsymbol{X}+\sum k_{H i}\left(\boldsymbol{I}_{n}-\boldsymbol{J}^{+} \boldsymbol{J}\right) \boldsymbol{\nabla} \boldsymbol{H}_{\boldsymbol{i}}
$$

In the case of the CDPR CoGiRo with the manipulator SIA20F on-board its mobile platform, the Jacobian matrix $\boldsymbol{J}$ is computed from the thirteen homogeneous transformation matrices defining the position and orientation of joint frame $j$ relative to frame $R_{0}$ :

$$
{ }^{0} \boldsymbol{T}_{j}=\left[\begin{array}{cccc}
\boldsymbol{s}_{j} & \boldsymbol{n}_{j} & \boldsymbol{a}_{j} & \boldsymbol{P}_{j} \\
0 & 0 & 0 & 1
\end{array}\right]
$$

Then, this Jacobian matrix can be written as: 


$$
\boldsymbol{J}=\left[\begin{array}{llll}
\boldsymbol{J}_{p 1} & \boldsymbol{J}_{p 2} & \ldots & \boldsymbol{J}_{p 1} \\
\boldsymbol{J}_{o 1} & \boldsymbol{J}_{o 2} & \ldots & \boldsymbol{J}_{o 13}
\end{array}\right]
$$

with $\boldsymbol{J}_{p j}=\sigma_{j} \boldsymbol{a}_{j}+\bar{\sigma}_{J}\left(\boldsymbol{a}_{j} \times\left(\boldsymbol{P}_{14}-\boldsymbol{P}_{j}\right)\right)$ and $\boldsymbol{J}_{o j}=\bar{\sigma}_{j} \boldsymbol{a}_{j}$, where $\sigma_{j}=1$ for a prismatic joint and $\sigma_{j}=0$ for a revolute joint, and where $\boldsymbol{P}_{14}$ is the position vector of the frame attached to the end-effector.

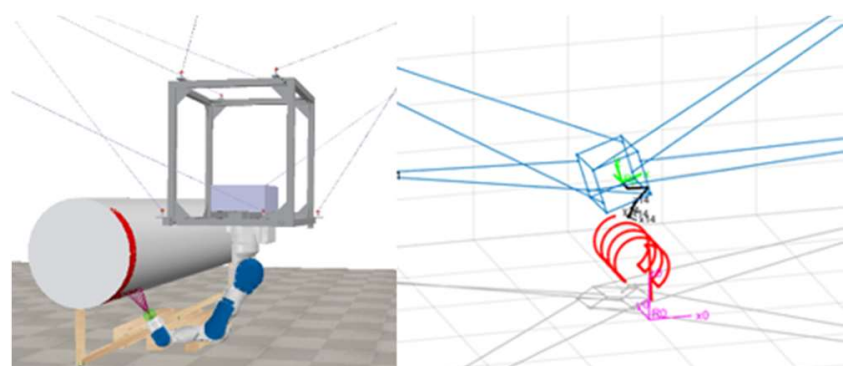

Fig. 3. Painting scenario (left); Painting path in red, CoGiRo in blue and SIA20F in black (right).

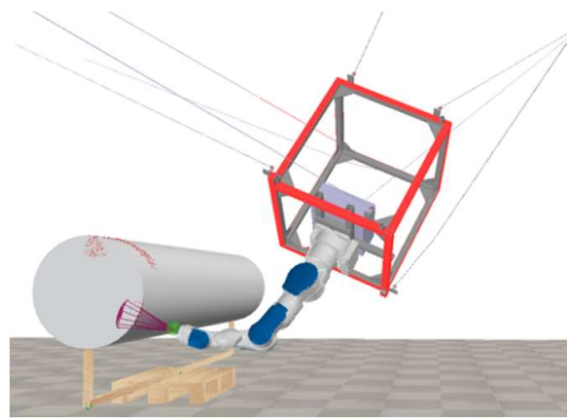

Fig. 4. Motion without optimization term: A cable-platform collision occurs.

\section{Simulation and Experimental Results}

In order to test the kinematic redundancy resolution method of Section 4, we consider a scenario where the manipulator on-board the CDPR paints a large cylinder, the latter representing for example a plane fuselage (Fig. 3).

In a first step, the equation $d \boldsymbol{Q}=\boldsymbol{J}^{+} d \boldsymbol{X}$ (without any optimization term) is used to find values for all joint coordinates to follow the desired path around the cylinder. The CoGiRo mobile platform orientation reaches large values which are not feasible on the real robot due to a cable colliding with the platform (Fig. 4). Equation (7) is then used, adding a first optimization term aiming at maintaining a platform orientation close to zero (Fig. 5). The cost functions $H_{o i}$ used for that purpose are described in the appendix. 


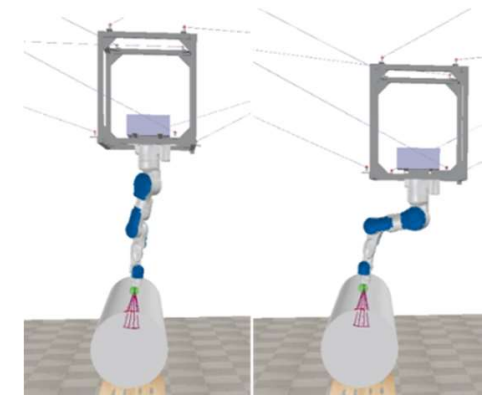

Fig. 5. Motion with an optimization of the platform orientation (left) and a further optimization to limit the platform height (right).

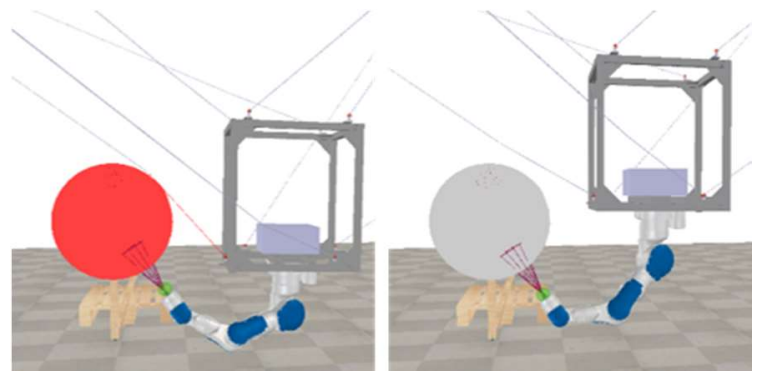

Fig. 6. Motion without (left) / with (right) optimization cost functions avoiding cablecylinder collisions.

With this optimization of the platform orientation, the CDPR platform reaches high positions along the vertical $\mathrm{z}$ axis at some points along the path (Fig. 5). These positions are not feasible because of the limitations on the cable tensions, the latter being too large for positions high in the workspace. Therefore, a second cost function $H_{z}$ (see the second paragraph of the appendix) is added to the local optimization in order to avoid overshooting a given limit on the CDPR platform vertical position (Fig. 5).

At this point however, some cables collide with the cylinder when painting the underside of the cylinder (Fig. 6). Adding a third set of cost functions $H_{d i}$ dealing with the distance between the cables and the cylinder (third paragraph of the appendix) allows the method to generate joint coordinate trajectories which avoid cable-cylinder collisions (Fig. 6).

To avoid singularities, a manipulability optimization term is finally introduced as presented in the fourth paragraph of the appendix. This term significantly changes the HCDPR behavior as can be seen in Fig. 7. The aim is to maximize the cost function $H_{m}=\operatorname{det}\left[\boldsymbol{J J}^{T}\right]$ (Fig. 8). Being given the hybrid nature of the considered robotic system (serial/parallel), the interpretation and relevance of using this manipulability optimization term deserves further investigations in future works. 


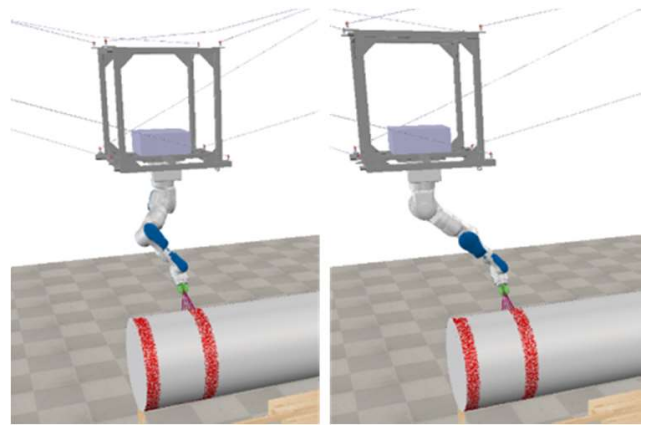

Fig. 7. Motion without (left) / with (right) manipulability optimization.

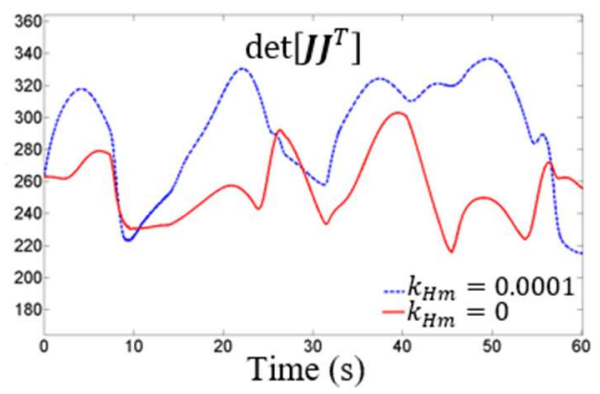

Fig. 8. Cost function $H_{m}=\operatorname{det}\left[\boldsymbol{J J}^{T}\right]$ along the desired trajectory.
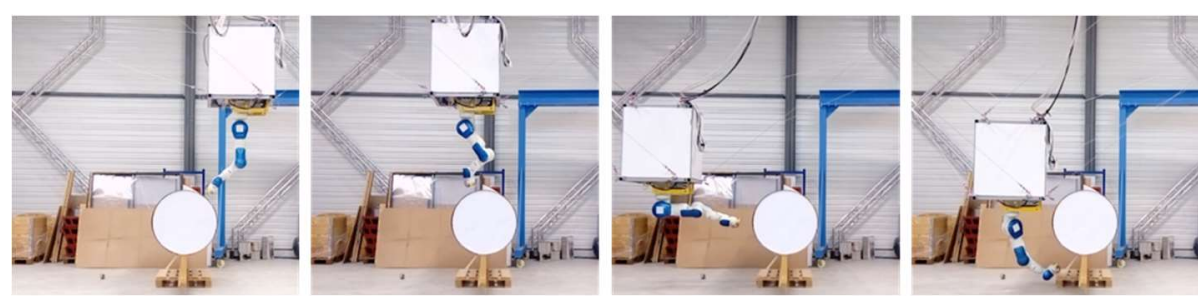

Fig. 9. CDPR CoGiRo and manipulator SIA20F following the painting path around a cylinder.

A complete motion avoiding collisions, with feasible platform orientation, limited platform height, and manipulability optimization avoiding singularities while remaining into the limits of the manipulator joint angles is finally obtained. This final motion has been tested on the physical HCDPR testbed which consists of the CDPR CoGiRo equipped with the SIA20F manipulator. For this purpose, a motion synchronization strategy has been set up. This synchronization is necessary since the two robots operate on different PLCs. It is based on sending on-the-fly target points to both systems (CDPR and manipulator), which was one of the requirements for choosing the manipulator (see in Section 2). A discretized path is used on both robots with common indexes on the points composing the path. The SIA20F goes through the points, reporting the actual index to the CDPR CoGiRo, which speeds up and slows down every step by 
reducing (respectively increasing) the time shift before the next step in order to keep up with the SIA20F. In the end, with a $2 \mathrm{~ms}$ discretized trajectory in input for the SIA20F and $20 \mathrm{~ms}$ for CoGiRo, the maximum latency between the two robots is $60 \mathrm{~ms}$. Some HCDPR configurations attained during the motion around the cylinder are shown in Fig. 9. The full demonstration video of this motion is provided in [21] and, with further explanations, in [22].

\section{Conclusion}

This paper reported the use of standard kinematic redundancy resolution techniques based on local optimizations on a HCDPR. The six-DOF CDPR CoGiRo equipped with a seven-DOF industrial manipulator was used to demonstrate the capability of such a hybrid parallel/serial robotic system to follow a path defined along the surface of a large-dimension cylinder, imitating the painting of a large object such as an airplane fuselage part. In order to successfully follow this path, several null space projections were used to achieve secondary tasks related to cable collision avoidance, manipulability optimization, and workspace limitations. Among several possible future works, the inclusion of wrench-feasibility constraints into the kinematic redundancy resolution and the consideration of the HCDPR dynamics could be relevant. Methods ensuring that problem constraints remain verified, which is not the case with null space projection techniques, would also be relevant.

\section{$7 \quad$ Acknowledgment}

This work was supported by the ANR under grant ANR-15-CE10-0006, project DexterWide

\section{Appendices}

\section{Cost functions related to the platform orientation}

To maintain the platform orientation close to zero, the following cost functions are considered:

$$
H_{o 1}=Q_{4}^{2} \quad H_{o 2}=Q_{5}^{2} \quad H_{o 3}=Q_{6}^{2}
$$

where $Q_{4}, Q_{5}$ and $Q_{6}$ are the fourth, fifth and sixth components of the generalized joint coordinate vector $\boldsymbol{Q}$ (Euler angles). Minimizing the cost functions $H_{o i}$ is achieved by assigning the same value $k_{H o}=-1$ to the corresponding three terms in Eq. (8). The cost functions $H_{o i}$ are minimized when orientation angles are null.

\section{Platform height cost function}

To avoid too high vertical positions of the CDPR mobile platform, the following cost function is used, where $Q_{1 d}$ is the desired platform height:

$$
H_{z}=\left(Q_{1}-Q_{1 d}-3\right)^{2}
$$

With a positive coefficient $k_{H z}$, the gradient $\boldsymbol{\nabla} \boldsymbol{H}_{\boldsymbol{z}}$ keeps the platform away from an 
upper limit situated at 3 meters above the ground (base frame $R_{0}$ ). The coefficient $k_{H z}$ is calculated as $\left.k_{H z}=e^{(-2} d\right)$ where $d$ is the distance between the current position of the platform reference point and the upper limit at 3 meters above the ground. Coefficient $k_{\mathrm{Hz}}$ increases exponentially from zero to one as $d$ decreases. The repulsive effect of the ceiling become noticeable when $d<0.2 \mathrm{~m}$.

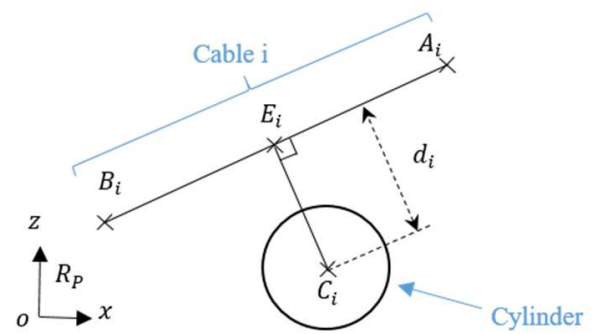

Fig. 10. Cable-cylinder distance

\section{Cable distance cost function}

The computation of the gradient $\boldsymbol{\nabla} \boldsymbol{H}_{\boldsymbol{d i}}$ of the cable-cylinder distance cost function $H_{d i}$ is done as follows. A cable is considered to be a straight-line segment $A_{i} B_{i}, A_{i}$ being the drawing point of the cable and $B_{i}$ its anchor point on the platform (Fig. 10). Sagging is thus neglected. All coordinates are expressed with respect to the frame $R_{P}$ whose $y$ axis is parallel to the cylinder axis. Then, the squared distance $d_{i}^{2}$ is used as the cost function, $H_{d i}=d_{i}^{2}$, where $d_{i}$ is given by:

$$
d_{i}=\left\|\overrightarrow{E_{l} C_{l}}\right\|=\sqrt{\left(x_{C i}-x_{E i}\right)^{2}+\left(z_{C i}-z_{E i}\right)^{2}}
$$

As illustrated in Fig. 10, the location of point $C_{i}$ is known and the location of point $E_{i}$ is computed as follows:

$$
\begin{aligned}
& x_{E i}=x_{B i}+\gamma_{i}\left(x_{A i}-x_{B i}\right) \\
& z_{E i}=z_{B i}+\gamma_{i}\left(z_{A i}-z_{B i}\right)
\end{aligned}
$$

Finding the location of $E_{i}$ is equivalent to finding $\gamma_{i}$ such as $d^{2}$ is minimal which is the case when:

$$
\frac{\partial d_{i}^{2}}{\partial \gamma_{i}}=0
$$

By using Eq. (13), (14) and (15), $\gamma_{i}$ can be computed which leads to $d_{i}^{2}$ and then to the gradient $\boldsymbol{\nabla} \boldsymbol{H}_{\boldsymbol{d i}}$. With a positive coefficient $k_{H d i}$, the cable-cylinder distance cost function keeps away each cable from the cylinder. The coefficient $k_{H d i}$ is calculated in the same way as $k_{H z}$, but relatively to $d_{i}$.

\section{Manipulability cost function}

To increase the manipulability and avoid singular configurations of the on-board manipulator, the following cost function is considered:

$$
H_{m}=\operatorname{det}\left[\boldsymbol{J} \boldsymbol{J}^{T}\right]
$$

In this case, the gradient is obtained by numerical derivation. Thus, the gradient: 


$$
\boldsymbol{\nabla} \boldsymbol{H}_{\boldsymbol{m}}=\left[\frac{\partial \operatorname{det}\left[\boldsymbol{J J}^{T}\right]}{\partial Q_{1}} \ldots \frac{\partial \operatorname{det}\left[\boldsymbol{J J}^{T}\right]}{\partial Q_{13}}\right]
$$

is replaced by:

$$
\boldsymbol{\nabla} \boldsymbol{H}_{\boldsymbol{m}}=\left[\frac{\operatorname{det}\left[\boldsymbol{J}_{\varepsilon_{1}} J_{\varepsilon_{1}}^{T}\right]-\operatorname{det}\left[J^{T}\right]}{\varepsilon_{1}} \ldots \frac{\operatorname{det}\left[\boldsymbol{J}_{\varepsilon_{13}} J_{\varepsilon_{13}}^{T}\right]-\operatorname{det}\left[J^{T}\right]}{\varepsilon_{13}}\right]
$$

where:

$$
\begin{gathered}
J_{\varepsilon_{1}}=J\left(Q_{c}+\varepsilon_{1}\right) \\
\vdots \\
J_{\varepsilon_{13}}=J\left(Q_{c}+\varepsilon_{13}\right)
\end{gathered}
$$

and:

$$
\begin{gathered}
\varepsilon_{1}=\left[\begin{array}{lllllllllllll}
\varepsilon & 0 & 0 & 0 & 0 & 0 & 0 & 0 & 0 & 0 & 0 & 0 & 0
\end{array}\right] \\
\vdots \\
\varepsilon_{13}=\left[\begin{array}{llllllllllll}
0 & 0 & 0 & 0 & 0 & 0 & 0 & 0 & 0 & 0 & 0 & 0
\end{array}\right]
\end{gathered}
$$

$\varepsilon$ is a small increment in the joint coordinate which has been tuned at $1.10^{-6}$. With a positive coefficient $k_{H m}=0.0001$, when used in Eq. (8), this gradient allows the manipulator to stay away from singular configurations.

\section{References}

[1] J. S. Albus, R. V. Bostelman, N. G. Dagalakis, The NIST ROBOCRANE, Journal of Robotic Systems, 10(15), 709-724 (1993).

[2] B. Duan, Y.-Y. Qiu, F. Zhang, B. Zi, On design and experiment of the feed cablesuspended structure for super antenna, Mechatronics, 19(14), 503-509 (2009).

[3] M. Gouttefarde, J.-F. Collard, N. Riehl, C. Baradat, Geometry Selection of a Redundantly Actuated Cable-Suspended Parallel Robot, IEEE Transaction on Robotics, 31(12), 501-510 (2015).

[4] C. Lambert, M. Nahon, D. Chalmers, Implementation of an Aerostat Positioning System With Cable Control, IEEE/ASME Transactions on Mechatronics, 12(11), 32-40 (2007).

[5] J.-P. Merlet, D. Daney, A portable, modular parallel wire crane for rescue operations, IEEE International Conference on Robotics and Automation, Anchorage, CA; USA (2010).

[6] A. Pott, H. Mütherich, W. Kraus, V. Schmidt, P. Miermeister, A. Verl, IPAnema: A family of Cable-Driven Parallel Robots for Industrial Applications, Cable-Driven Parallel Robots, Springer, 119-134 (2013).

[7] O. Tempier, P.-E. Hervé, M. Gouttefarde, Cable-driven parallel robot CoGiRo moving a robot manipulator, [Online]. Available: https://youtu.be/-CmjXwXEZJA (2015).

[8] IRT Jules Verne, Cable-driven parallel robot equipped with a UR10 manipulator, [Online]. Available: https://youtu.be/J3R01IgwUi8 (2018).

[9] J. Albus, Modular Suspended Platform. US Patent 6,566,834 (2003).

[10] T. Arai, S. Matsumura, Y. Yoshimura, H. Osumi, A proposal for a wire suspended manipulator, Robotica, 17, 3-9 (1999). 
[11] A. Pott et al, Cable-Driven Parallel Robot IPAnema 2 at Automatica 2010. [Online]. Available: https://youtu.be/9-qQYv90_Og (2010).

[12] S. Lefrançois, C. Gosselin, Point-to-Point Motion Control of a Pendulum-like 3-Dof Underactuated, IEEE International Conference on Robotics and Automation, Anchorage, USA (2010).

[13] M. Bamdad, F. Taheri, N. Abtahi, Dynamic Analysis of a Hybrid Cable-Suspended Planar, IEEE International Conference on Robotics and Automation, Seattle, Washington (2015).

[14] H. Osumi, Y. K. M. Utsugi, Development of a Manipulator Suspended by Parallel Wire Structure, IEEE/RSJ International Conference Intelligent Robotic Systems, Takamatsu, Japan, 2000.

[15] M. Gouttefarde, Static Analysis of Planar 3-DOF Cable-Suspended Parallel Robots Carrying a Serial Manipulator, 6th European Conference on Mechanism Science (EUCOMES), Nantes, France (2016).

[16] R. Qi, M. Rushton, A. Khajepour, W. W. Melek, Decoupled modeling and model predictive control of a hybrid cable-driven robot, Robotics and Autonomous Systems, 118 (2019).

[17] D. Bury, J.-B. Izard, M. Gouttefarde, F. Lamiraux, Continuous Collision Detection for a Robotic Arm Mounted on a Cable-Driven Parallel Robot, IEEE/RSJ International Conference on Intelligent Robots and Systems (IROS), Macau, China (2019).

[18] D. Bury, J.-B. Izard, M. Gouttefarde, F. Lamiraux, Continuous Tension Validation for Cable-Driven Parallel Robots, IEEE/RSJ International Conference on Intelligent Robots and Systems (IROS), Las Vegas, United States (2020).

[19] J. Mirabel, S. Tonneau, P. Fernbach, A.-K. Seppala, M. Campana, N. Mansard, F. Lamiraux, HPP: a new software for constrained motion planning, IEEE/RSJ Intelligent Robots and Systems (IROS), Daejeon, Korea (2016).

[20] M. Gouttefarde et al, CoGiRo: A large-dimension cable-driven parallel robot, [Online]. Available: https://youtu.be/2b4YwFZhtIE (2012).

[21] O. Tempier, M. Michelin, P.-E. Hervé, M. Gouttefarde, J.-B. Izard, Cable robot CoGiRo and Yaskawa SIA20F: Path Following -- ANR project DexterWide. [Online]. Available: https://youtu.be/g0_OqK-ZWdU (2019).

[22] M. Michelin, O. Tempier, P.-E. Hervé, M. Gouttefarde, J.-B. Izard, Cable-driven parallel robot CoGiRo equipped with a robot arm Yaskawa SIA20F. [Online] Available: https://youtu.be/UKbbWN43UcQ (2021).

[23] Xamla, 2017-07-07 SDA10D Joystick Control. [Online] Available: https://youtu.be/KgzYKmDy8jk (2018).

[24] ANR, Project DexterWide. [Online] Available: https://www.lirmm.fr/dexterwide (2019).

[25] W. Khalil, E. Dombre, Modeling, Identification and Control of Robots, London: Hermes Penton (2002)

[26] B. Siciliano, O. Khatib, (Eds) Springer Handbook of Robotics, Chap. 11, Springer (2008).

[27] M. Gouttefarde, J. Lamaury, C. Reichert, T. Bruckmann, A Versatile Tension Distribution Algorithm for n-DOF Parallel Robots Driven by n+2 Cables, IEEE Transactions on Robotics, 31(6), 1444-1457 (2015). 